\title{
Diabetic Retinopathy Treatment
}

\author{
a report by \\ Fernando López, ${ }^{1}$ María Rodríguez,1 Joaquín Marticorena ${ }^{2,3}$ and Francisco Gómez-Ulla ${ }^{1,3}$
}

1. Ophthalmology Department, University Hospital Complex of Santiago de Compostela; 2. Saint Paul's Eye Unit, Royal Liverpool University Hospital;

3. Technological Institute of Ophthalmology, Santiago de Compostela

DOI: 10.17925/EOR.2007.00.00.68

Diabetic retinopathy (DR) is the main cause of blindness in industrialised countries in patients aged between 20 and 64 years, and is the cause of $10 \%$ of new blindness cases every year. ${ }^{1}$ Tight control of systemic factors, photocoagulation and vitrectomy are the main strategies against the DR; however, recently several new treatments have been developed.

Control of Systemic Factors

\section{Glycaemic Control}

The most effective medical treatment to slow the progression of DR is glycaemic control. The Diabetic Control and Complications Trial (DCCT) was designed to compare the effects of conventional with intensive diabetes therapy in the development and progression of early microvascular complications. This study demonstrated that intensive therapy reduced the adjusted mean risk of retinopathy by 76 and $54 \%$ in insulin-dependent diabetes mellitus (IDDM) patients with no DR and with very mild to moderate non-proliferative $D R$, respectively. Mean glycosylated haemoglobin $\left(\mathrm{HbA}_{1 \mathrm{c}}\right)$ was the dominant predictor of $\mathrm{DR}$ progression. The recommendation that arose from this trial was to maintain glycaemic status as close to the normal range as possible, as well as early implementation and long-term maintenance of intensive therapy in IDDM patients. ${ }^{2}$ In patients with type 2 diabetes, intensive treatment is also recommended with significantly lower levels of $\mathrm{HbA}_{1 \mathrm{c}}$ and microvascular end-points. ${ }^{3}$

\section{Blood Pressure Control}

Management of blood pressure should have a high priority in the treatment of type 2 diabetes. Tight blood pressure control has been proved to lead to a $37 \%$ reduction in the risk of microvascular disease. ${ }^{4}$

\section{Serum Lipid Control}

The Wisconsin Epidemiologic Study of Diabetic Retinopathy (WESDR) and the Early Treatment Diabetic Retinopathy Study (ETDRS) showed evidence that elevated serum lipids increase the severity of DR and retinal hard exudates.5,6 Hydroxymethilglutaryl coenzyme A (HMG CoA) inhibitors could be useful in the management of DR and diabetic macular oedema (DMO) in patients with dyslipidaemia, but further studies are necessary to demonstrate the effects of statins.

\section{Laser Photocoagulation}

Timely photocoagulation with Argon laser remains the principal therapy for sight-threatening DR. Laser techniques can be classified as pan-retinal, focal or grid photocoagulation. The Diabetic Retinopathy Study (DRS) and the ETDRS are the two major trials providing strong support for the therapeutic benefit of photocoagulation. In the first trial, scatter panretinal photocoagulation (PRP) demonstrated a reduction of the risk of severe loss of vision $(<5 / 200)$ after two years in patients with high-risk PDR (eyes with new vessels and pre-retinal or vitreous haemorrhage; eyes with new vessels on or within one disc diameter of the optic disc equalling or exceeding one-quarter to one-third disc area in extent, even in the absence of pre-retinal or vitreous haemorrhage). ${ }^{7}$ In eyes with severe nonproliferative $D R$ or non-high-risk proliferative $D R$, the treatment is recommended under certain circumstances (i.e. rapid advance of retinal disease, history of poor patient follow-up). The ETDRS showed that early PRP reduces the risk of progression of retinopathy, the risk of vitrectomy and the risk of severe visual loss. ${ }^{8}$ The ETDRS also demonstrated that PRP is not recommended for eyes with mild or moderate non-proliferative disease. The adverse effects of PRP are moderate visual loss and visual field loss, which may be evident in the months immediately after photocoagulation. In eyes with clinical significant macular oedema (CSMO) as defined by the ETDRS, the recommended treatment is focal laser photocoagulation. This modality has demonstrated the reduction of the risk of moderate decrease of visual acuity (VA) by $50 \%$ in a period of three years. However, the definition of CSMO does not distinguish between focal or diffuse diabetic macula oedema. In eyes with diffuse oedema a grid laser photocoagulation can be performed in the macular area avoiding the fovea.

\section{Pars Plana Vitrectomy}

Complications of severe proliferative DR-like tractional retinal detachment, combined tractional/rhegmatogenous detachment, severe vitreous haemorrhage and recurrent vitreous haemorrhage requires treatment with pars plana vitrectomy (PPV). In patients with proliferative DR and severe vitreous haemorrhage, PPV is indicated. Early PPV (within six months) has demonstrated to be of benefit in preserving VA in those patients with VA $\geq 20 / 400$ in the presence of severe proliferative DR (neovacularisation, fibrous proliferation) or severe vitreous haemorrhage in IDDM patients, especially if diabetes duration is less than 20 years, but not in type ॥ diabetic patients. ${ }^{9}$ The latter must be interpreted in the context of new instruments and surgical techniques developed in the last few years, such as bi-manual surgery with delamination/segmentation, new light sources, perfluorocarbon liquid and 25G $23 \mathrm{G}$ vitrectomy. PPV also allows the completion of PRP intra-operatively after the removal of the vitreous haemorrhage and clearing of the media. Diabetic macula oedema in the presence of vitreomacular traction signs is another indication of PPV..$^{10} \mathrm{PPV}$ is also necessary for the treatment of complications secondary to an initial PPV, such as recurrent intraocular bleeding, anterior fibrovascular proliferation or retinal detachment.

Pharmacological vitreolisis with autologous plasmin and human recombinant microplasmin have been studied in different experimental models. These agents may be a useful adjunct to conventional vitreous surgery for DMO by inducing posterior vitreous detachment. A clinical trial to evaluate the efficacy and safety of microplasmin in patients in whom vitrectomy is indicated is ongoing. 
Potential Pharmacological Therapies

\section{Corticosteroids}

Corticosteroids are known to reduce vascular permeability, reduce blood-retinal barrier breakdown, downregulate vascular endothelial growth factor (VEGF) production and inhibit certain matrix metalloproteinases. Recently, intravitreal triamcinolone acetonide (IVTA) injection has been widely used as an off-label therapy for DMO. In a prospective, randomised, controlled clinical trial, Gillies et al. observed that those eyes treated with repeated IVTA presented better VA and less macular oedema compared with those that received placebo after two years of follow-up. ${ }^{11}$ In patients with refractory diffuse macular oedema, IVTA has been shown to achieve a short-term decrease in macular thickness measured with OCT. ${ }^{12}$ Injection of IVTA has also been used as an adjuvant for macular grid laser, observing a maintained VA improvement for six months. ${ }^{13}$ The role of IVTA as an adjuvant treatment to PRP has also been evaluated with promising results, reducing neovascularisation and macular thickness in 35 patients. ${ }^{14}$ Like other glucocorticoids, IVTA is related to some secondary effects such as a rise in intraocular pressure, development of cataracts and endophthalmitis. The main drawback of IVTA is the temporary effect of the steroid, the need for repeated injections with doses between 2 and $25 \mathrm{mg}$ and the risk associated with each procedure. Recently, new corticosteroid treatments have been developed as intravitreal systems capable of delivering a small but constant dose of the drug. A fluocinolone acetonide implant (Retisert, Bausch \& Lomb, Rochester) has been approved for clinical use in patients with uveitis. Recently, an update of the multicentre, randomised clinical trial for the treatment of DMO revealed significantly less macular oedema and better VA than standard care after three years of implantation. ${ }^{15}$

\section{Vascular Endothelial Growth Factor Inhibitors}

VEGF is produced by the pigment epithelial cells, pericytes and endothelial cells of the retina in response to hypoxia from capillary loss and/or microaneurysm formation, and is a key mediator of angiogenesis and blood-retina barrier breakdown in the ischaemic retina. ${ }^{16}$ Clinical studies have revealed that intravitreal VEGF concentration increased in subjects as they progressed from non-proliferative DR to active PDR. ${ }^{17,18}$ Similarly, successful PRP reduced intraocular VEGF levels by $75 \%$ in patients treated for ocular neovascularisation. ${ }^{19}$ This suggests that specific inhibition of VEGF activity may prevent retinal neovascularisation and associated blood flow abnormalities. The follow-up of the studies is still short to make any specific treatment recommendations; however, the results appear promising, as we will comment below. In human clinical trials, the intravitreally administered aptamer VEGF inhibitor pegaptanib sodium (Macugen, Eyetech Pharmaceuticals, Inc., New York) has shown its benefit in a phase II trial involving DMO. ${ }^{20} \mathrm{~A}$ phase III clinical trial is ongoing. ${ }^{21}$ The Macugen Diabetic Retinopathy Study Group found that most subjects assigned to pegaptanib showed regression of retinal neovascularisation by week 36.22 Ranibizumab (Lucentis, Genentech, Inc., South San Francisco) is a fully humanised monoclonal anti-VEGF-A Fab fragment developed specifically for ophthalmic applications. ${ }^{23}$ Ranibizumab was designed to bind all biologically active forms of VEGF-A. In a pilot study, Chun et al. observed that ranibizumab therapy has the potential to maintain or improve VA and reduce retinal thickness in patients with DMO. ${ }^{24}$ Bevacizumab (Avastin, Genentech Inc., San Francisco) is a complete fulllength humanised antibody that binds to all subtypes of VEGF. The results from the Pan-American Collaborative Retina Study Group at six-month follow-up showed that primary intravitreal bevacizumab at doses of
$1.25-2.5 \mathrm{mg}$ seem to provide stability or improvement in $\mathrm{VA}$, retinal thickness and fluorescein angiography pattern in patients with DMO. ${ }^{25}$ It has also been associated with rapid regression of retinal and iris neovascularisation secondary to diabetes mellitus. Bevacizumab's role in such treatment is limited by the short duration of the effect. However, bevacizumab as an adjunctive treatment to PRP may have better results and may minimise the latter's propensity to exacerbate the macular oedema. ${ }^{26}$ Bevacizumab has been also used as a pre-vitrectomy adjunct for the repair of tractional retinal detachment in eyes with severe active proliferative DR. ${ }^{27}$

\section{Protein Kinase C Inhibitors}

Protein kinase C (PKC) is a family of 13 enzymes, of which the $\beta$ isoform has been the most closely linked to the development of diabetic microvascular complications. It is induced by high glucose due to an increased diacylglycerol (DAG) level, and activation of PKC- $\beta$ appears to mediate increases in retinal vascular permeability and neovascularisation in animal models and changes in retinal blood flow in diabetic patients. PKC activation is important in the intracellular signalling of VEGF, which is hypothesised to be a principal mediator of retinal neovascularisation and permeability in diabetes. ${ }^{28-30}$ Ruboxistaurin (RBX-LI333531) is a PKC- $\beta$ isozyme-selective inhibitor that is orally bioavailable and has been shown to ameliorate the adverse effects of high glucose in a number of animal models of diabetic microvascular complications. The PKC-probable phospholipid-transporting ATPase (PKC-DRS2) Group ${ }^{31}$ showed that patients with moderately severe to severe non-proliferative DR treated with ruboxistaurin had 40\% lower risk of sustained moderate visual loss and less progression of macular oedema and needed less laser treatment than the control group. PKC412 (another PKC inhibitor) has been related to a significant decrease of macular oedema and improvement of VA in diabetic subjects. ${ }^{32}$

\section{Somatostatin Agonists}

It has been observed that the progression of proliferative DR accelerates during puberty, when the tissue sensitivity to growth hormone $(\mathrm{GH})$ is increased, there are few diabetic patients with pituitary infiltrative lesions and GH-deficient dwarfs have no evidence of macro- or microvascular disease. Somatostatin analogues may inhibit angiogenesis directly through somatostatin receptors of endothelial cells, and indirectly through the inhibition of post-receptor signalling events of peptide growth factor such as insulin-like growth factor (IGF-1) and VEGF. Octreotide - a somatostatin analogue and IGF-1 antagonist - decreased the need for PRP compared with conventional treatment in patients with severe non-proliferative $D R$ or early proliferative $D R$, but the incidence of progression to severe proliferative DR was similar in both groups. ${ }^{33} \mathrm{~A}$ clinical trial using the pegvisomant - a GH-receptor antagonist - did not demonstrate any effect on DR. ${ }^{34}$

\section{Cyclooxygenase-2 Inhibitors}

Cyclooxygenase (COX)-2 is an enzyme that causes angiogenesis through prostanoid - primarily prostaglandin E2 synthesis - in response to inflammation. A recent study found COX-2 was expressed more in diabetic than in non-diabetic subjects. ${ }^{35} \mathrm{~A}$ human trial to evaluate the effects of the COX-2 inhibitor celecoxib (Celebrex, Pfizer, New York) on proliferative diabetic retinopathy is currently recruiting patients.

\section{Interferon- $\alpha 2 a$}

Interferon- $\alpha 2 \mathrm{a}$ is a peptide that influences gene expression and protein synthesis, causing inhibition of vascular endothelial proliferation and migration in the laboratory. A pilot study that included patients with 


\section{Retina}

active diabetic neovascularisation suggested that interferon- $\alpha 2 a$ may limit progression of neovascularisation in DR. ${ }^{36}$ Nevertheless, other trials have showed disappointing results, as well as a high incidence of side effects. ${ }^{37-39}$ Future large studies with interferon- $\alpha-2$ a may be feasible.

\section{Angiotensin-converting Enzyme Inhibitors/Angiotensin II Receptor Blockers}

There is evidence that the renin-angiotensin system is expressed in the eye. Studies have shown that angiotensin-converting enzyme (ACE) is produced locally by vascular endothelial cells and retinal pigment epithelial cells. ${ }^{40,41}$ Furthermore, ACE expression has been reported to have adverse effects on retinal blood flow and vascular structures that are independent of changes in systemic blood pressure. ${ }^{42}$ The Diabetes Incidence after REnal Transplantation (DIRECT) study evaluating the effects of candesartan (angiotensin II receptor blocker) on DR is ongoing. ${ }^{43}$

\section{Antioxidants}

Diabetes mellitus may cause reactive oxygen species (ROS), which may activate aldose reductase, PKC and diacylglycerol (DAG) formation. In addition, ROS stimulates VEGF production in a dose-dependent manner. All these products may play a role in the pathogenesis of vascular complications, both microvascular and macrovascular, and an early marker of such damage is the development of endothelial dysfunction. ${ }^{44}$ However, the role of oxidative stress in diabetes is questioned by the uneven results of the intervention studies. ${ }^{45-47}$

\section{Conclusions}

It is essential to emphasise to every diabetic patient the importance of an steady metabolism and tight blood pressure control in the prevention of DR. Laser treatment is indicated once DR is manifested and threatening the VA because of the presence of macular oedema or new vessels. Laser photocoagulation and PPV can reduce the risk of further loss of vision, but are not exempt from adverse effects and complications. New therapies are being developed as single or adjuvant tools to treat advanced stages of PDR or macular oedema. The better understanding of DR has opened many pathways for new pharmacological therapies. Ongoing and future clinical trials will determine the capacity for preventing the presence and/or progression of DR and the safety of these emerging treatments.
1. Pesce A, De Santiago F, Epidemiologia de la Retinopatía diabética. In: Alfaro V, Gómez-Ulla F, Quiroz-Mercado H, Figueroa MS (eds), Retinopatía diabética, Tratado médicoquirúrgico, Barcelona:Mac Line, 2007;35-42.

2. The Diabetes Control and Complications Trial/Epidemiology of Diabetes Interventions and Complications Research Group, Retinopathy and nephropathy in patients with type 1 diabetes four years after a trial of intensive therapy, $N$ Engl I Med 2000,342:381-9.

3. UK Prospective Diabetes Study Group, Intensive blood-glucose control with sulphonylureas or insulin compared with conventional treatment and risk of complications in patients with type 2 diabetes, UKPDS 33, Lancet, 1998;352:837-53.

4. UK Prospective Diabetes Study Group, Tight blood pressure control and risk of macrovascular and microvascular complications in type 2 diabetes, UKPDS 38, BMJ, 1998;317:703-13.

5. Klein BE, et al., The Wisconsin Epidemiologic Study of Diabetic Retinopathy. XIII. Relationship of serum cholesterol to retinopathy and hard exudate, Ophthalmology, 1991;98:1261-5.

6. Chew EY, Klein ML, Ferris FL III, et al., Association of elevated serum lipid levels with retinal hard exudate in diabetic retinopathy. Early Treatment Diabetic Retinopathy Study (ETDRS) Report 22, Arch Ophthalmol, 1996;114:1079-84.

7. Diabetic Retinopathy Study, Report Number 6. Design, methods, and baseline results. Report Number 7. A modification of the Airlie House classification of diabetic retinopathy, Invest Ophthalmol Vis Sci, 1981:21:1-226.

8. The Early Treatment Diabetic Retinopathy Study Research Group, Early Treatment Diabetic Retinopathy Study design and baseline patient characteristics. ETDRS report number 7, Ophthalmology, 1991;98:741-56.

9. The Diabetic Retinopathy Vitrectomy Study Research Group, Early vitrectomy for severe proliferative diabetic retinopathy in eyes with useful vision. Results of a randomized trial - Diabetic Retinopathy Vitrectomy Study Report 3, Ophthalmology, 1988;95:1307-20.

10. Lewis H, Abrams GW, Blumenkranz MS, Campo RV, Vitrectomy for diabetic macular traction and edema associated with posterior hyaloidal traction, Ophthalmology, 1992;5:759.

11. Gillies MC, Sutter FK, Simpson JM, et al., Intravitreal triamcinolone for refractory diabetic macular edema: two-year results of a double-masked, placebo-controlled, randomized clinical trial, Ophthalmology, 2006;113:1533-8.

12. Audren F, Erginay A, et al., Intravitreal triamcinolone acetonide for diffuse diabetic macular oedema: 6-month results of a prospective controlled trial, Acta Ophthalmol Scand, 2006;84:624-30.

13. Kang SW, Sa HS, et al., Macular grid photocoagulation after intravitreal triamcinolone acetonide for diffuse diabetic macular edema, Arch Ophthalmol, 2006;124:653-8.

14. Bandello F, Polito A, et al., Triamcinolone as adjunctive treatment to laser panretinal photocoagulation for proliferative diabetic retinopathy, Arch Ophthalmol, 2006;124:643-50.
15. Pearson P, Levy B, Comstock T, Fluocinolone Acetonide Implant Study Group. Fluocinolone acetonide intravitreal implant to treat diabetic macular edema: 3-year results of a multicenter clinical trial, Invest Ophthalmol Vis Sci, 2006;47:\$5442.

16. Aiello $L P$, Avery RL, et al., Vascular endothelial growth factor in ocular fluid of patients with diabetic retinopathy and other retinal disorders, N Engl J Med, 1994;331:1480-87.

17. Aiello $L P$, Avery RL, et al., Vascular endothelial growth factor in ocular fluid of patients with diabetic retinopathy and other retinal disorders, N Engl J Med, 1994;331:1480-87.

18. Miller JW, Vascular endothelial growth factor and ocular neovascularization, Am J Pathol, 1997;151:13-23.

19. Aiello $L P$, Avery RL, et al., Vascular endothelial growth factor in ocular fluid of patients with diabetic retinopathy and other retinal disorders, N Engl J Med, 1994;331:1480-87.

20. Cunningham ET Jr, Adamis AP, Altaweel M, et al., A phase II randomized double-masked trial of pegaptanib, an anti-vascular endothelial growth factor aptamer, for diabetic macular edema, Ophthalmology, 2005;112:1747-57.

21. Shams $N$, et al., Role of vascular endothelial growth factor in ocular angiogenesis, Ophthalmol Clin North Am, 2006;19:335-44.

22. Adamis AP, Altaweel M, Bressler NM, et al., Changes in retinal neovascularization after pegaptanib (Macugen) therapy in diabetic individuals, Ophthalmology, 2006;113:23-8.

23. Presta $L G$, et al., Humanization of an anti-vascular endothelial growth factor monoclonal antibody for the therapy of solid tumors and other disorders, Cancer Res, 1997:57:4593-9.

24. Chun DW, Heier JS, Topping TM, et al., A pilot study of multiple intravitreal injections of ranibizumab in patients with centerinvolving clinically significant diabetic macular edema, Ophthalmology, 2006;113:1706-12.

25. Arevalo JF, Fromow-Guerra J, Quiroz-Mercado H, et al., Primary intravitreal bevacizumab (Avastin) for diabetic macular edema: results from the Pan-American Collaborative Retina Study Group at 6-month follow-up, Ophthalmology, 2007;114:743-50.

26. Avery RL, Pearlman J, Pieramici DJ, et al., Intravitreal bevacizumab (Avastin) in the treatment of proliferative diabetic retinopathy, Ophtha/mology, 2006;113:1695-1715.

27. Chen $E$, et al., Use of intravitreal bevacizumab as a preoperative adjunct for tractional retinal detachment repair in severe proliferative diabetic retinopathy, Retina, 2006;26:699-700.

28. Aiello LP, Bursell SE, Clermont $A$, Vascular endothelial growth factor-induced retinal permeability is mediated by protein kinase $C$ in vivo and suppressed by an orally effective-isoformselective inhibitor, Diabetes, 1997;46:1473-80.

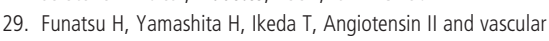
endothelial growth factor in the vitreous fluid of patients with diabetic macular edema and other retinal disorders, $\mathrm{Am} J$ Ophthalmol, 2002;133:537-43.

30. Suzuma K, Takahara N, Suzuma I, et al., Characterization of protein kinase $\mathrm{C}$ isoform's action on retinoblastoma protein phosphorylation, vascular endothelial growth factor-induced endothelial cell proliferation, and retinal neovascularization, Proc Nat Acad Sci, 2002;99:721-6.

31. Aiello $L P$, et al., Effect of ruboxistaurin on visual loss in patients with diabetic retinopathy, Ophtha/mology, 2006;113:2221-30.

32. Campochiaro PA, Reduction of diabetic macular edema by oral administration of the kinase inhibitor PKC412, Invest Ophthalmol Vis Sci, 2004;45:922-31.

33. Grant MB, et al., The efficacy of octreotide in the therapy of severe nonproliferative and early proliferative diabetic retinopathy: a randomized controlled study, Diabetes Care, 2000:23:504-9.

34. Growth Hormone Antagonist for Proliferative Diabetic Retinopathy Study Group, The effect of a growth hormone receptor antagonist drug on proliferative diabetic retinopathy, Ophthalmology, 2001;108:2266-72.

35. Sennlaub F, Valamanesh F, Vazquez-Tello A, et al., Cyclooxygenase-2 in human and experimental ischemic proliferative retinopathy, Circulation, 2003;108:198-204.

36. Leibovitch I, et al., Interferon alpha-2a for proliferative diabetic retinopathy after complete laser panretinal photocoagulation treatment, Ophtha/mic Surg Lasers Imaging, 2004;35:16-22.

37. Jaakkola $A$, et al., Interferon alpha-2a in the treatment of exudative senile macular degeneration, Acta Ophtha/mol, 1994;72:545-9.

38. Thomas MA, Ibanez HE, Interferon alfa-2a in the treatment of subfoveal choroidal neovascularization, Am I Ophthalmol, 1993;115:563-8.

39. Thoelen A, Menozzi M, Huber C, Messmer E, Treatment of choroidal neovascularization in age-related macular degeneration with interferon alpha-2a: a short term, nonrandomized pilot study, Ger J Ophthalmol, 1995;4:137-43.

40. Danser AH, Derkx FH, Admiraal PJ, et al., Angiotensin levels in the eye, Invest Ophthalmol Vis Sci, 1994;35:1008-18.

41. Wagner J, Jan, et al., Demonstration of renin mRNA, angiotensinogen $\mathrm{mRNA}$, and angiotensin converting enzyme mRNA expression in the human eye: evidence for an intraocular renin-angiotensin system, Br J Ophthalmol, 1996;80:159-63.

42. Engler $\mathrm{CB}$, et al., Blood-retina barrier permeability in diabetes during acute ACE-inhibition, Acta Ophthalmol, 1991;69:581-5.

43. Sjolie AK, Porta M, Parving HH, et al., The Diabetic Retinopathy Candesartan Trials (DIRECT) Programme: baseline characteristics, J Renin Angiotensin Aldosterone Syst, 2005;6:25-32.

44. Giugliano D, Ceriello A, Paolisso G, Oxidative stress and diabetic vascular complications, Diabetes Care, 1996;19:257-67.

45. Marchioli R, Schweiger C, Levantesi G, et al., Antioxidant vitamins and prevention of cardiovascular disease: epidemiological and clinical trial data, Lipids, 2001;36:S53-63.

46. Millen $A E$, Gruber $M$, Klein $R$, et al., Relations of serum ascorbic acid and alpha-tocopherol to diabetic retinopathy in the Third National Health and Nutrition Examination Survey, Am J Epidemiol, 2003;158:225-33.

47. Bursell SE, et al., High-dose vitamin E supplementation normalizes retinal blood flow and creatinine clearance in patients with type 1 diabetes, Diabetes Care, 1999;22:1245-51. 